\title{
Sarcopenic obesity is associated with macroalbuminuria in patients with type 2 diabetes: a cross-sectional study
}

\author{
Fuyuko Takahashi, Yoshitaka Hashimoto, Ayumi Kaji, Ryosuke Sakai, Takuro Okamura, \\ Masahide Hamaguchi and Michiaki Fukui
}

Department of Endocrinology and Metabolism, Kyoto Prefectural University of Medicine, Graduate School of Medical Science, Kyoto 621-8585, Japan

\begin{abstract}
Sarcopenia is associated with the risk of albuminuria in patients with type 2 diabetes mellitus (T2DM), and obesity is a risk factor for proteinuria. However, the association between sarcopenic obesity and diabetic nephropathy, including albuminuria, in patients with T2DM has not been reported. The study included 206 men and 163 women with T2DM who participated in the KAMOGAWA-DM cohort, which investigating the natural history of diabetes since 2014. Sarcopenia was defined as having both low skeletal muscle mass index (SMI, $\left.\mathrm{kg} / \mathrm{m}^{2}\right)\left(<7.0 \mathrm{~kg} / \mathrm{m}^{2}\right.$ for men and $<5.7 \mathrm{~kg} / \mathrm{m}^{2}$ for women) and low handgrip strength ( $<28 \mathrm{~kg}$ for men and $<18 \mathrm{~kg}$ for women). Obesity was diagnosed by the percentage of body fat ( $>30 \%$ for men and $>35 \%$ for women). The patient was said to have sarcopenic obesity if he/she had both sarcopenia and obesity. Urinary albumin excretion of patients with sarcopenic obesity was higher than that of patients without sarcopenic obesity (median [interquartile range]: 342.0 [41.8-467.5] vs. 21.0 [9.0-75.4] mg/g Cr, $p=0.016$ ). Additionally, sarcopenic obesity was associated with the presence of macroalbuminuria, compared with non-sarcopenic obesity (adjusted odds ratio 6.92 [95\% confidence interval:1.63-29.4], $p=0.009$ ). Adjusted odds ratios of sarcopenic obesity, sarcopenia only, and obesity only for the presence of macroalbuminuria were $6.52(1.47-28.8, p=0.014), 1.29(0.45-3.71, p=0.638)$, and $0.78(0.38-1.58, p=$ 0.482 ), respectively, compared with neither sarcopenia nor obesity. This study indicated that sarcopenic obesity is associated with albuminuria, especially macroalbuminuria, in Japanese patients with T2DM.
\end{abstract}

Key words: Muscle, Nephropathy, Obese, Sarcopenia, Type 2 diabetes

NUMBER OF PATIENTS, especially elderly patients, with type 2 diabetes mellitus (T2DM) is increasing globally and they often develop sarcopenia [1]. Sarcopenia is defined as the age-associated decline of muscle strength, mass, and function [2]. Sarcopenia is now known as a risk factor for cardiovascular disease (CVD) and mortality [3-5]. Additionally, it has been reported that the presence of obesity in combination with sarcopenia, called as sarcopenic obesity, is a greater risk factor for mortality than the sarcopenia alone [6, 7]. It has been reported that there is a possibility that T2DM has a high risk of sarcopenic obesity $[8,9]$.

The prevalence of diabetic nephropathy, which is the major cause of end stage renal disease (ESRD), is also globally increasing [10]. Albuminuria, especially macro-

Submitted Oct. 19, 2020; Accepted Feb. 8, 2021 as EJ20-0655

Released online in J-STAGE as advance publication Mar. 17, 2021

Correspondence to: Yoshitaka Hashimoto, MD, PhD, Department of Endocrinology and Metabolism, Kyoto Prefectural University of Medicine, Graduate School of Medical Science, 465, Kajii cho, Kamigyo-ku, Kyoto-city, Kyoto 621-8585, Japan.

E-mail: y-hashi@koto.kpu-m.ac.jp albuminuria, is an independent risk factor not only for incident ESRD, [11-13] but also for mortality [14], particularly in patients with diabetes. Recent studies have showed that sarcopenia is associated with the risk of albuminuria in patients with T2DM $[15,16]$, and obesity is a risk factor for proteinuria [17]. Thus, there is a possibility that sarcopenic obesity is associated with albuminuria in patients with T2DM. However, there has not been a study that explored this association. Therefore, the present cross-sectional study researched the relationship between sarcopenic obesity and diabetic nephropathy, including the presence of macroalbuminuria, in patients with T2DM.

\section{Materials and Methods}

\section{Study participants}

The KAMOGAWA-DM cohort study is an ongoing cohort study to elucidate the natural history of diabetes [18]. This study has been carried out since 2014. Patients enrolled in this study provided written informed consent. The present study is a part of KAMOGAWA-DM study. 
It includes the outpatients visiting the Department of Endocrinology and Metabolism, Kyoto Prefectural University of Medicine (KPUM) Hospital (Kyoto, Japan), and the Department of Diabetology, Kameoka Municipal Hospital (Kameoka, Japan). The present study included patients with T2DM who underwent bioimpedance analysis (BIA) between 15 January 2016 and 17 April 2018 [19]. The patients with unreliable BIA data, lacking urinary albumin excretion, and with poor handgrip strength were excluded from the study. This study was approved by the KPUM Ethics Committee (approval number RBMR-E-466-6) and has been conducted in accordance with the principles of Declaration of Helsinki.

\section{Data collection}

We gave a standardized questionnaire to all participants, and categorized them as smokers or non-smokers, and non-exercisers or regular exercisers based on their responses in the questionnaire. Further, venous blood was gathered from the participants who had fasted overnight and the levels of triglycerides, high-density lipoprotein (HDL) cholesterol, creatinine $(\mathrm{Cr})$, uric acid, and fasting plasma glucose were measured. Estimated glomerular filtration rate (eGFR) was calculated using the equation of the Japanese Society of Nephrology, i.e., $\mathrm{eGFR}=194 \times \mathrm{Cr}^{-1.094} \times \operatorname{age}^{-0.287}\left(\mathrm{~mL} / \mathrm{min} / 1.73 \mathrm{~m}^{2}\right)$ $(\times 0.739$, if woman) [20]. The hemoglobin A1c (HbA1c) level was estimated using high-performance liquid chromatography and was expressed as a National Glycohemoglobin Standardization Program unit. Further, the immunoturbidimetric method was used for the evaluation of urinary albumin excretion (UAE), which was calculated using the formula: UAE $(\mathrm{mg} / \mathrm{g} \mathrm{Cr})=$ urinary albumin concentration $(\mathrm{mg} / \mathrm{L}) /$ urinary $\mathrm{Cr}$ concentration $(\mathrm{g} / \mathrm{L})$. The mean UAE value was estimated from three independent readings. The stage of diabetic nephropathy was defined as follows: stage 1 , eGFR $\geq 30 \mathrm{~mL} / \mathrm{min} / 1.73 \mathrm{~m}^{2}$ and normoalbuminuria (UAE $<30 \mathrm{mg} / \mathrm{g} \mathrm{Cr}$ ); stage 2, eGFR $\geq 30 \mathrm{~mL} / \mathrm{min} / 1.73 \mathrm{~m}^{2}$ and microalbuminuria (UAE 30$300 \mathrm{mg} / \mathrm{g} \mathrm{Cr}$ ); stage 3 , eGFR $\geq 30 \mathrm{~mL} / \mathrm{min} / 1.73 \mathrm{~m}^{2}$ and macroalbuminuria (UAE $>300 \mathrm{mg} / \mathrm{g} \mathrm{Cr}$ ); and stage 4, eGFR $<30 \mathrm{~mL} / \mathrm{min} / 1.73 \mathrm{~m}^{2}$ [21]. None of the patients required maintenance dialysis during this study. Additionally, the data for medications, including those for diabetes and hypertension (renin-angiotensin-aldosterone [RAS] inhibitor), and dyslipidemia (statin) were obtained. Data for medications were obtained from the patients' medical records.

Body composition was evaluated using a multifrequency impedance body composition analyzer, InBody 720 (InBody Japan, Tokyo, Japan) [19]. The data for body weight $(\mathrm{kg})$, appendicular muscle mass $(\mathrm{kg})$, and body fat mass $(\mathrm{kg})$ were obtained. Body mass index
(BMI, $\mathrm{kg} / \mathrm{m}^{2}$ ) and skeletal muscle mass index (SMI, $\mathrm{kg} / \mathrm{m}^{2}$ ) were calculated by dividing body weight $(\mathrm{kg})$ and appendicular muscle mass $(\mathrm{kg})$, respectively, with the square of height $(\mathrm{m})$. The percent body fat mass (\%) was calculated by dividing body fat mass $(\mathrm{kg})(\times 100)$ with body weight $(\mathrm{kg})$. The handgrip strength was measured twice for each hand using a handgrip dynamometer (Smedley, Takei Scientific Instruments Co., Ltd., Niigata, Japan) and the maximum value was included in the analyses [19]. Sarcopenia was diagnosed by low SMI and low handgrip strength [22]. The cut-off values for low handgrip strength were $<28 \mathrm{~kg} / \mathrm{m}^{2}$ for men and $<18 \mathrm{~kg} / \mathrm{m}^{2}$ for women, and those for low SMI were $<7.0 \mathrm{~kg} / \mathrm{m}^{2}$ for men and $<5.7 \mathrm{~kg} / \mathrm{m}^{2}$ for women [22]. Obesity was defined by the percentage of body fat, i.e., $>30 \%$ for men and $>35 \%$ for women [23]. The patient was said to have sarcopenic obesity if he/she had both sarcopenia and obesity [23].

\section{Statistical analyses}

The data are showed as median (1st quartile-3rd quartile median), mean (standard deviation [SD]), or frequencies of potential confounding variables. Patients were divided into the following four groups based on the absence or presence of sarcopenia and obesity: absence of both sarcopenia and obesity $(-/-)$, sarcopenia only $(+/-)$, obesity only $(-/+)$, and sarcopenic obesity $(+/+)$. The differences in the continuous variables were evaluated by the Mann-Whitney $U$ test, Kruskal-Wallis test and Steel-Dwass test, and one-way analysis of variance (ANOVA) and the Tukey-Kramer test, and those in the categorical variables were evaluated by the Chi-square test and the Holm test.

Further, we evaluated the association between sarcopenia and/or obesity, and albuminuria. Because UAE was a skewed variable, logarithmic transformation was done before performing multivariable linear regression analyses, which were performed to evaluate the association of combined effect of sarcopenia and/or obesity with $\log _{\mathrm{e}}(\mathrm{UAE}+1)$. Sex, age, smoking habit, exercise, BMI, systolic blood pressure, levels of HbA1c, uric acid, creatinine, duration of diabetes, consumption of RAS inhibitor, insulin, SGLT2 inhibitors, GLP-1 receptor agonists and statin were used for covariates.

Furthermore, we evaluated the effect of the presence of sarcopenia, obesity, and sarcopenic obesity on the presence of macroalbuminuria. Since the number of patients with macroalbuminuria was less, the propensity score, which preserves the statistical power by reducing covariates into a single variable, was used. The propensity score was calculated from multivariable logistic regression models that included age, sex, exercise, RAS inhibitor, insulin, SGLT2 inhibitors, GLP-1 receptor 
agonists, statin, duration of diabetes, family history of diabetes, smoking, systolic blood pressure, BMI, HbAlc, uric acid and creatinine. The c-statistic for propensity score model was 0.89 , which indicates an acceptable discrimination between the presence or absence of macroalbuminuria. Further, the odds ratios of sarcopenia, obesity, and sarcopenic obesity for the presence of macroalbuminuria were calculated using the propensity score. The odds ratios of the presence or absence of sarcopenia or obesity for the presence of macroalbuminuria were also calculated.

In addition, we also performed sub-analyses of using the cut-off of obesity of BMI $\geq 25 \mathrm{~kg} / \mathrm{m}^{2}$ [24] and evaluated the association between sarcopenia and/or obesity, and albuminuria, and the effect of the presence of sarcopenic obesity on the presence of macroalbuminuria.

The statistical analyses were conducted using JMP software ver. 13.2 (SAS Institute Inc., Cary, NC, USA) and EZR (Saitama Medical Center, Jichi Medical University, Saitama, Japan) [25], which is a graphical user interface for R (The R Foundation for Statistical Computing, Vienna, Austria). The differences with $p$ value $<0.05$ were considered statistically significant.

\section{Results}

In the present study, 383 patients $(212$ men and 171 women) with T2DM were extracted. Among them, 8 patients ( 5 men and 3 women) who had not undergone the multifrequency impedance body composition analyzer test, 4 patients (women) who had not undergone the handgrip strength test, and 2 patients with no date of UAE (1 man and 1 woman) were excluded from the study (Fig. 1). Finally, the study population comprised 369 patients.
The clinical characteristics of the participants based on the absence or presence of sarcopenia and/or obesity are shown in Table 1. Mean age of patients with nonsarcopenia/non-obesity, sarcopenia only, obesity only, and sarcopenic obesity was 67.0 (8.9), 75.0 (8.4), 63.2 (12.3), and 75.4 (10.0) years, respectively. The percentage of patients with sarcopenia only, obesity only, and sarcopenic obesity was 7.9\% $(n=29 / 369), 33.1 \%(n=$ $122 / 369)$, and $2.7 \%(n=10 / 369)$, respectively. The patients with sarcopenic obesity were significantly older than those with obesity only. The BMI of patients with sarcopenic obesity was higher than those with sarcopenia alone, and lower than those with obesity alone. The proportion of patients using SGLT2 inhibitors or GLP-1 receptor agonists was lower in patients with sarcopenia only than those with neither sarcopenia nor obesity. Fig. 2 shows the proportion of diabetic nephropathy based on the stages according to the absence or presence of sarcopenia and/or obesity. The sarcopenic obesity was associated with the higher stage of diabetic nephropathy $(p<$ 0.001).

Urinary albumin excretion of patients with sarcopenic obesity was higher than that of patients without sarcopenic obesity (median [interquartile range]: 342.0 [41.8467.5] vs. 21.0 [9.0-75.4] mg/g Cr, $p=0.016$ ). Table 2 shows the association of combined effect of sarcopenia and/or obesity with $\log _{\mathrm{e}}(\mathrm{UAE}+1)$. The patients with sarcopenic obesity had significantly higher $\log _{\mathrm{e}}$ (UAE +1 ) than those with neither sarcopenia nor obesity.

Furthermore, the sarcopenic obesity was associated with the presence of macroalbuminuria after adjusting for covariates (odds ratio 6.92 [95\% confidence interval: $1.63-29.4], p=0.009)$. Compared to the non-sarcopenia/ non-obesity patients, the patients with sarcopenic obesity had the highest risk for macroalbuminuria (OR 6.52

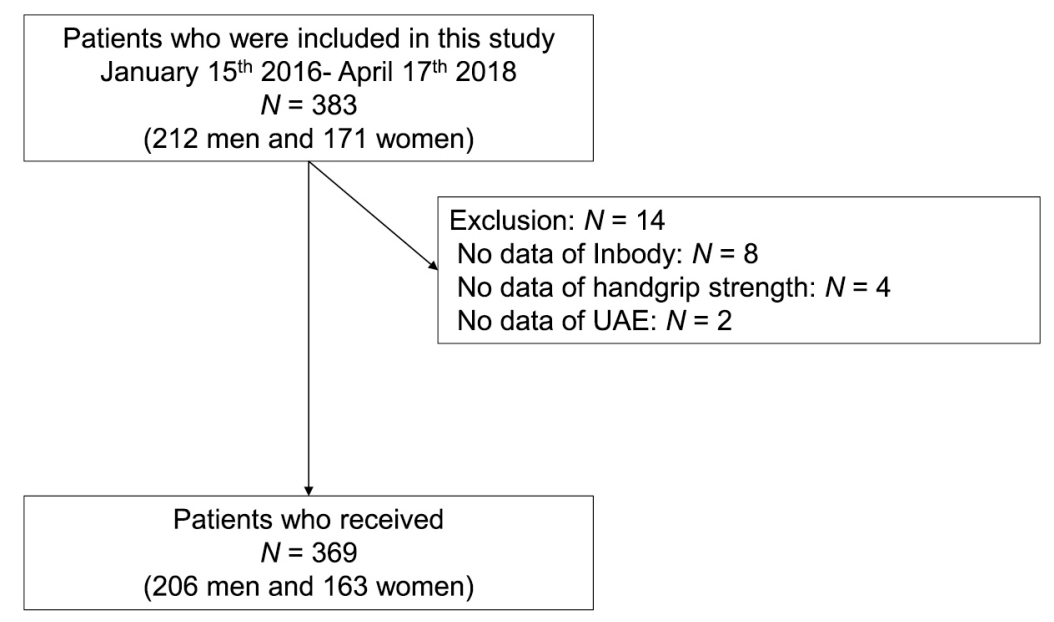

Fig. 1 Study flow diagram for the registration of patients.

UAE, urinary albumin excretion. 
Table 1 Clinical characteristics of study participants according to the presence or absence of sarcopenia or obesity

\begin{tabular}{|c|c|c|c|c|c|}
\hline Sarcopenia/obesity & $(-/-)$ & $(+/-)$ & $(-/+)$ & $(+/+)$ & $p$ \\
\hline Men/Women & $132 / 76$ & $22 / 7$ & $45 / 77$ & $7 / 3$ & $<0.001$ \\
\hline Age, years & $67.0(8.9)$ & $75.0(8.4)^{*}$ & $63.2(12.3)^{* \dagger}$ & $75.4(10.0)^{*}$ & $<0.001$ \\
\hline Duration of diabetes, years & $14.5(10.1)$ & $23.7(10.8)^{*}$ & $10.6(8.0)^{* \dagger}$ & $15.0(10.5)$ & $<0.001$ \\
\hline Family history of diabetes $(-/+)$ & 109/99 & $20 / 9$ & $65 / 57$ & $8 / 2$ & 0.138 \\
\hline Height, cm & $163.0(8.8)$ & $159.2(6.4)$ & $158.3(9.5)^{*}$ & $157.5(7.8)$ & $<0.001$ \\
\hline Body weight, kg & $60.5(10.1)$ & $51.8(6.6)^{*}$ & $72.0(13.7)^{* \dagger}$ & $62.5(6.7)^{\dagger}$ & $<0.001$ \\
\hline Body mass index, $\mathrm{kg} / \mathrm{m}^{2}$ & $22.7(2.5)$ & $20.4(1.8)^{*}$ & $28.7(4.5)^{* \dagger}$ & $25.3(2.6)^{\dagger+}$ & $<0.001$ \\
\hline Systolic blood pressure, $\mathrm{mmHg}$ & $133.5(19.7)$ & $132.2(15.2)$ & $135.3(16.7)$ & $132.1(25.0)$ & 0.781 \\
\hline Diastolic blood pressure, $\mathrm{mmHg}$ & $78.4(11.1)$ & $72.0(11.1)^{*}$ & $81.8(10.1)^{* \dagger}$ & $74.8(14.0)$ & $<0.001$ \\
\hline Insulin $(-/+)$ & $153 / 55$ & $18 / 11$ & $101 / 21$ & $9 / 1$ & 0.047 \\
\hline SGLT2 inhibitors $(-/+)$ & $184 / 24$ & $27 / 2 *$ & $87 / 35$ & $9 / 1$ & $<0.001$ \\
\hline GLP-1 receptor agonists $(-/+)$ & $185 / 23$ & $26 / 3^{*}$ & $90 / 32$ & $8 / 2$ & 0.003 \\
\hline RAS inhibitor $(-/+)$ & $119 / 89$ & $13 / 16$ & $66 / 56$ & $6 / 4$ & 0.625 \\
\hline Statin $(-/+)$ & $129 / 79$ & $18 / 11$ & $75 / 47$ & $7 / 3$ & 0.963 \\
\hline Smoking $(-/+)$ & $173 / 35$ & $26 / 3$ & $106 / 16$ & $10 / 0$ & 0.367 \\
\hline Habitual alcohol consumption $(-/+)$ & $181 / 27$ & $27 / 2$ & $112 / 10$ & $10 / 0$ & 0.308 \\
\hline Habit of exercise $(-/+)$ & $108 / 100$ & $15 / 14$ & $65 / 57$ & $4 / 6$ & 0.883 \\
\hline Hemoglobin A1c, \% & $7.3(1.2)$ & $7.4(1.0)$ & $7.5(1.5)$ & $7.3(0.7)$ & 0.757 \\
\hline Hemoglobin A1c, mmol/mol & $56.4(13.4)$ & $57.7(11.1)$ & $58.2(16.1)$ & $56.7(7.8)$ & 0.757 \\
\hline Plasma glucose, $\mathrm{mmol} / \mathrm{L}$ & $8.2(2.7)$ & $8.6(2.2)$ & $8.3(3.0)$ & $7.6(1.8)$ & 0.811 \\
\hline Creatinine, umol/L & $74.8(31.9)$ & $69.6(19.4)$ & $69.2(26.4)$ & $88.1(47.5)$ & 0.128 \\
\hline $\mathrm{eGFR}, \mathrm{mL} / \mathrm{min} / 1.73 \mathrm{~m}^{2}$ & $68.6(17.2)$ & $73.6(22.1)$ & $71.5(20.3)$ & $63.2(28.5)$ & 0.255 \\
\hline Uric acid, mmol/L & $301.5(76.3)$ & $300.5(76.7)$ & $311.6(69.4)$ & $362.2(85.2)$ & 0.064 \\
\hline $\mathrm{TG}, \mathrm{mmol} / \mathrm{L}$ & $1.4(0.9)$ & $1.1(0.6)$ & $1.8(1.0)^{* \dagger}$ & $1.5(0.7)$ & $<0.001$ \\
\hline HDL cholesterol, mmol/L & $1.6(0.5)$ & $1.7(0.5)$ & $1.5(0.4)$ & $1.4(0.3)$ & 0.029 \\
\hline Urinary albumin excretion, $\mathrm{mg} / \mathrm{gCr}$ & $20.0(8.0-70.0)$ & $19.8(12.0-110.9)$ & $27.1(11.0-79.2)$ & $342.0(41.8-467.5)$ & 0.028 \\
\hline Diabetic nephropathy stage $(1 / 2 / 3 / 4)$ & $125 / 56 / 24 / 3$ & $16 / 8 / 5 / 0$ & $68 / 40 / 10 / 4$ & $2 / 1 / 5 / 2 * \dagger$ & $<0.001$ \\
\hline Handgrip strength, kg & $29.8(9.0)$ & $21.8(5.6)^{*}$ & $27.2(9.5)^{\dagger}$ & $19.6(5.2)^{* \hbar}$ & $<0.001$ \\
\hline Appendicular muscle mass, $\mathrm{kg}$ & $18.9(4.1)$ & $15.7(2.8)^{*}$ & $18.1(4.3)^{\dagger}$ & $15.5(2.7)^{*}$ & $<0.001$ \\
\hline Body fat mass, $\mathrm{kg}$ & $14.8(4.3)$ & $12.4(3.6)$ & $28.3(8.0)^{* \dagger}$ & $23.6(4.6)^{* \dagger}$ & $<0.001$ \\
\hline Percent body fat mass, $\%$ & $24.4(5.9)$ & $23.6(5.4)$ & $39.1(6.0)^{* \dagger}$ & $37.8(6.3)^{* \dagger}$ & $<0.001$ \\
\hline SMI, $\mathrm{kg} / \mathrm{m}^{2}$ & $7.0(1.0)$ & $6.2(0.7)^{*}$ & $7.1(1.1)^{\dagger}$ & $6.2(0.6)^{* \ddagger}$ & $<0.001$ \\
\hline
\end{tabular}

Data was expressed as mean (standard deviation), median (interquartile range) or number. The difference between group was evaluated by ANOVA, Kruskal-Wallis test or chi-square test. RAS, renin-angiotensin system; eGFR, estimated glomerular filtration rate; TG, triglycerides; HDL, high-density lipoprotein; SMI, skeletal muscle mass index. ${ }^{*}, p<0.05 v s .(-/-){ }^{\dagger}, p<0.05 v s .(+/-)$; and ${ }^{\star}, p<0.05 v s$. $(-1+)$.

[95\% CI: 1.47-28.80], $p=0.014$; Table 3). Further, the adjusted odds ratios of sarcopenic obesity, sarcopenia only, and obesity only for the presence of macroalbuminuria were $6.52(95 \% \mathrm{CI}: 1.47-28.8, p=0.014), 1.29$ ( $95 \%$ CI: $0.45-3.71, p=0.638)$, and 0.78 (95\% CI: 0.38 $1.58, p=0.482$ ), respectively. Additionally, the patients with sarcopenic obesity were at higher risk of macroalbuminuria compared to the patients with sarcopenia only (OR 5.06 [95\% CI: 0.94-27.2], $p=0.059$ ) and obesity only (OR 8.41 [95\% CI: 1.85-38.2], $p=0.006$ ).

The results of sub-analysis of the association between sarcopenia and/or obesity, and $\log _{\mathrm{e}}(\mathrm{UAE}+1)$, using the 
Table 2 The association of sarcopenic obesity with $\log _{\mathrm{e}}$ (urinary albumin excretion +1 )

\begin{tabular}{lccccc}
\hline Sarcopenia/obesity & $(-/-)$ & $(+/-)$ & $(-/+)$ & $(+/+)$ & $p$ \\
\hline Model 1 & $3.36(3.14-3.59)$ & $3.68(3.08-4.28)$ & $3.56(3.27-3.85)$ & $4.99(3.97-6.01)^{* \dagger}$ & 0.018 \\
Model 2 & $3.31(3.09-3.54)$ & $3.40(2.78-4.01)$ & $3.68(3.38-3.98)$ & $4.72(3.70-5.74)^{*}$ & 0.022 \\
Model 3 & $3.63(3.28-3.98)$ & $3.85(3.22-4.48)$ & $3.88(3.53-4.23)$ & $5.03(4.09-5.97)^{*}$ & $<0.001$ \\
\hline
\end{tabular}

Values for outcome variables are geometric means and 95\% CI. Log, logarithms.

Model 1 was unadjusted model, model 2 was adjusted for age and sex and model 3 was adjusted for age, sex, smoking, exercise, duration of diabetes, renin-angiotensin system inhibitor, insulin, SGLT2 inhibitors, GLP-1 receptor agonists, statin, systolic blood pressure, body mass index, HbA1c, uric acid, creatinine. ${ }^{*}, p<0.05 v s .(-/-)$; and ${ }^{\dagger}, p<0.05 v s .(-/+)$.

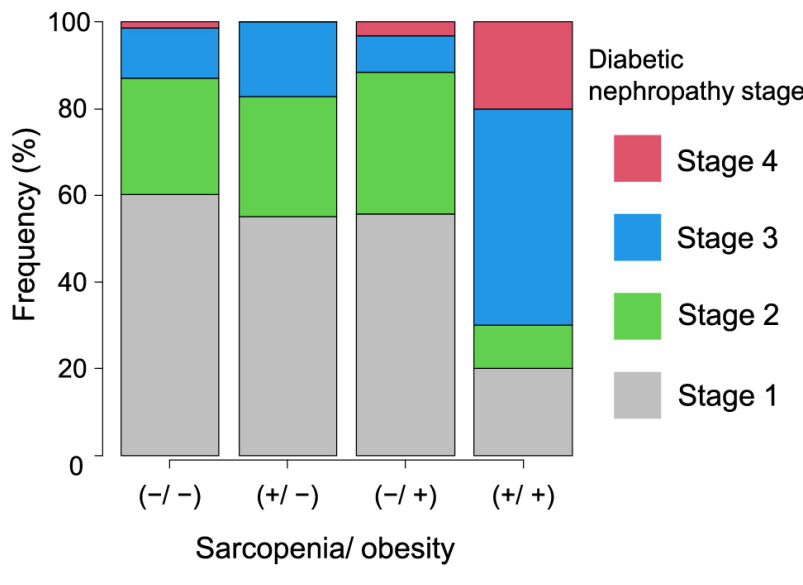

Fig. 2 Proportion of diabetic nephropathy stage according to the presence/absence of sarcopenia and obesity

cut-off of obesity of BMI $\geq 25 \mathrm{~kg} / \mathrm{m}^{2}$, are show in Table 4. The proportion of sarcopenia alone, obesity alone and sarcopenic obesity were $9.2 \%(n=34), 36.3 \%(n=134)$ and $1.4 \%(n=5)$, respectively. The patients with sarcopenic obesity had significantly higher $\log _{\mathrm{e}}(\mathrm{UAE}+1)$ than those with neither sarcopenia nor obesity. Moreover, the sarcopenic obesity, using the cut-off of obesity of BMI $\geq 25 \mathrm{~kg} / \mathrm{m}^{2}$, was associated with the presence of macroalbuminuria after adjusting for covariates (OR 19.90 [95\% CI: 2.03-194.00], $p=0.010$ ) (Table 5).

\section{Discussion}

The present study researched the relationship between sarcopenic obesity and albuminuria in patients with T2DM. The proportion of patients with sarcopenia only and sarcopenic obesity were $7.9 \%$ and $2.7 \%$, respectively. These proportions were similar to the previous studies reported [26, 27]. The UAE of patients with sarcopenic obesity was higher than that of patients neither with sarcopenia and obesity. Additionally, sarcopenic obesity was associated with the presence of macroalbuminuria in patients with T2DM.

Sarcopenia has been established as a risk factor for CVD and mortality [3-5]. Additionally, it is known to be
Table 3 Odds ratio of the presence or absence of sarcopenia or obesity for presence of macroalbuminuria

\begin{tabular}{lcc}
\hline & OR $(95 \% \mathrm{CI})$ & $p$ \\
\hline Absence of sarcopenia & ref & - \\
Presence of sarcopenia & $2.23(0.97-5.09)$ & 0.058 \\
\hline Absence of obesity & ref & - \\
\hline Presence of obesity & $0.94(0.50-1.78)$ & 0.894 \\
\hline Absence of sarcopenic obesity & ref & - \\
\hline Presence of sarcopenic obesity & $6.92(1.63-29.4)$ & 0.009 \\
\hline Presence/absence of sarcopenia and obesity & \\
\hline$(-/-)$ & ref & - \\
\hline$(+/-)$ & $1.29(0.45-3.71)$ & 0.638 \\
$(-/+)$ & $0.78(0.38-1.58)$ & 0.482 \\
$(+/+)$ & $6.52(1.47-28.8)$ & 0.014 \\
\hline
\end{tabular}

Since the cases of macroalbuminuria is not enough. Propensity score was used for covariates. Propensity score was evaluated by multivariable logistic regression models that include the age, sex, duration of diabetes, family history of diabetes, smoking, exercise, renin-angiotensin system inhibitor, insulin, SGLT2 inhibitors, GLP-1 receptor agonists, statin, systolic blood pressure, body mass index, HbA1c, uric acid, and creatinine. The c-statistic for propensity score model was 0.89 .

a risk of albuminuria [15, 16]. Sarcopenic obesity, which more likely causes lifestyle-related morbidities than obesity without sarcopenia, may lead to a further reduction in mobility and is associated with higher mortality rates than sarcopenia alone [6, 7]. Moreover, a recent study showed that sarcopenic obesity had a higher risk of albuminuria than sarcopenia [28]. However, the definition of sarcopenia used in that study was SMI (\%), which is the appendicular muscle mass $(\mathrm{kg})$ divided by body weight $(\mathrm{kg}) \times 100$, which was different from SMI $\left(\mathrm{kg} / \mathrm{m}^{2}\right)$ used in our study. In fact, SMI $(\%)$ and SMI $\left(\mathrm{kg} / \mathrm{m}^{2}\right)$ are clinically different markers [29]. In the present study, the risk of albuminuria was the highest in the patients with sarcopenic obesity. Previous studies showed that both sarcopenia and obesity were the risk of macroalbuminuria [15-17]. On the other hand, in this study, we revealed 
Table 4 The association of sarcopenic obesity, using BMI cut-off, with $\log _{\mathrm{e}}$ (urinary albumin excretion +1 )

\begin{tabular}{lccccc}
\hline Sarcopenia/obesity & $(-/-)$ & $(+/-)$ & $(-/+)$ & $(+/+)$ & $p$ \\
\hline Model 1 & $3.31(3.08-3.54)$ & $3.79(3.24-4.34)$ & $3.62(3.35-3.90)$ & $5.53(4.09-6.96)^{*}$ & 0.007 \\
Model 2 & $3.25(3.03-3.48)$ & $3.49(2.92-4.06)$ & $3.75(3.46-4.03)^{*}$ & $5.28(3.85-6.70)^{*}$ & 0.004 \\
Model 3 & $3.57(3.21-3.92)$ & $3.91(3.32-4.51)$ & $3.88(3.55-4.22)$ & $5.44(4.17-6.71)^{*}$ & $<0.001$ \\
\hline
\end{tabular}

Values for outcome variables are geometric means and 95\% CI. Log, logarithms. Obesity was defined as BMI $\geq 25 \mathrm{~kg} / \mathrm{m}^{2}$.

Model 1 was unadjusted model, model 2 was adjusted for age and sex and model 3 was adjusted for age, sex, smoking, exercise, duration of diabetes, renin-angiotensin system inhibitor, insulin, SGLT2 inhibitors, GLP-1 receptor agonists, statin, systolic blood pressure, body mass index, HbAlc, uric acid, creatinine. *, $p<0.05$ vs. $(-/-)$.

that not the presence of sarcopenia only or obesity only but the sarcopenic obesity was a higher risk of macroalbuminuria. Thus, we should focus on sarcopenic obesity for a higher risk of macroalbuminuria, which are higher risk of renal dysfunction progression.

The diagnostic criteria for sarcopenic obesity, especially regarding obesity, are not unified yet $[30,31]$. Percentage of body fat, BMI, and waist circumference has been used for definitions and diagnose for obesity [31]. In the present study, we used the cut-off values for percentage of body fat $>35 \%$ in women and $>30 \%$ in men for obesity [23]. A recent study has suggested that this criterion is suitable for the Japanese [23]. On the other hand, in the sub-analysis using the cut-off of obesity of BMI $\geq 25 \mathrm{~kg} / \mathrm{m}^{2}$, sarcopenic obesity was associated with albuminuria. Therefore, regardless of which definition is used, sarcopenic obesity was associated with albuminuria.

To prevent muscle mass loss, exercise and protein intake are important [32]. On the other hand, in obesity, it is important to reduce total calorie intake for weight loss [33]. Thus, there is a possibility that limiting carbohydrates and fats, but not reducing protein, might be needed in patients sarcopenic obesity [34]. A previous study revealed that protein intake was not associated with progression of albuminuria in patients with T2DM without macroalbuminuria [35]. However, it remained to be unclear regarding protein intake in diabetic nephropathy; and thus, further research on the prevention and improvement of sarcopenic obesity is needed.

The possible link between sarcopenic obesity and albuminuria can be explained as follows. The loss of skeletal muscle has a close association with insulin resistance, reactive oxygen species, and chronic inflammation, which can, in turn, promote albuminuria. Sarcopenia has been reported to be associated with an inflammatory state driven by cytokines and oxidative stress [36]. In fact, sarcopenia has been reported to be associated with nuclear factor $\kappa \mathrm{B}(\mathrm{NF}-\mathrm{\kappa B})$ and protein kinase $\mathrm{B}$ (Akt) signaling through secretion of interleukin-6 (IL-6), tumor necrosis factor- $\alpha$ (TNF- $\alpha$ ), and transforming growth factor- $\beta$ (TGF- $\beta$ ) [37]. Addi-
Table 5 Odds ratio of the presence or absence of sarcopenia or obesity, using BMI cut-off, for presence of macroalbuminuria

\begin{tabular}{lcc}
\hline $\begin{array}{l}\text { Presence/absence of sarcopenia } \\
\text { and obesity }\end{array}$ & OR $(95 \% \mathrm{CI})$ & $p$ \\
\hline$(-/-)$ & ref & - \\
$(+/-)$ & $1.41(0.52-3.78)$ & 0.498 \\
$(-/+)$ & $0.85(0.43-1.68)$ & 0.632 \\
$(+/+)$ & $19.90(2.03-194.00)$ & 0.010 \\
\hline
\end{tabular}

Since the cases of macroalbuminuria is not enough. Propensity score was used for covariates. Propensity score was evaluated by multivariable logistic regression models that include the age, sex, duration of diabetes, family history of diabetes, smoking, exercise, renin-angiotensin system inhibitor, insulin, SGLT2 inhibitors, GLP-1 receptor agonists, statin, systolic blood pressure, body mass index, HbA1c, uric acid, and creatinine. The c-statistic for propensity score model was 0.89 .

tionally, a previous study has revealed that obesity, like sarcopenia, increases albuminuria by triggering cascades of events including insulin resistance, increased reactive oxygen species, and chronic inflammation [38]. Obesity is also associated with Akt and NF- $\mathrm{KB}$ signaling through secretion of IL-6, TNF- $\alpha$, and TGF- $\beta$. Insulin resistance, reactive oxygen species, and chronic inflammation have been reported to induce albuminuria [39]. Therefore, having both sarcopenia and obesity accelerates these insulin resistance and chronic inflammation through fat accumulation in the muscles and insufficient working of insulin. Taken together, the aforementioned mechanisms suggest that the synergistic effect of sarcopenia and obesity is associated with albuminuria.

This study has a few limitations. First, the study design was cross-sectional. Therefore, the causal relationship between the sarcopenic obesity and albuminuria is unclear. Second, the generalizability of the results of the present study in non-Japanese T2DM patients is unclear. Third, we did not have the data of insulin resistance, reactive oxygen species, and inflammation, which might be link the sarcopenic obesity and albuminuria. 
Fourth, the sample size of this study was relatively small. The future prospects are to examine longitudinal albuminuria changes in patients with T2DM with sarcopenic obesity, and to clarify the association between sarcopenic obesity and albuminuria.

In conclusion, the findings of the present study suggest an association between sarcopenic obesity and albuminuria, including macroalbuminuria, in Japanese patients with T2DM. Preventing sarcopenic obesity would reduce the risk of diabetic nephropathy, which is a major cause of ESRD in T2DM.

\section{Acknowledgments}

We would like to thank Editage (www.editage.jp) for English language editing.

\section{Availability of Data and Materials}

The datasets used and/or analyzed during the current study are available from the corresponding author on reasonable request.

\section{Funding}

None.

\section{Disclosure}

Dr. Hashimoto reports grants from Asahi Kasei Pharma and personal fees from Sanofi K.K., Daiichi Sankyo Co. Ltd., Novo Nordisk Pharma Ltd., and Mitsubishi Tanabe Pharma Corp. outside the submitted work. Dr. Hamaguchi reports grants from Mitsubishi Tanabe Pharma Corp., Sanofi K.K., Asahi Kasei Pharma, Kyowa Kirin Co. Ltd., Eli Lilly Japan K.K., Astellas Pharma Inc., Sumitomo Dainippon Pharma Co. Ltd., Novo Nordisk Pharma Ltd., Takeda Pharma Co. Ltd, Nippon Boehringer Ingelheim Co. Ltd., and Daiichi Sankyo Co. Ltd. outside the submitted work. Prof. Fukui received grants from Taisho Pharma Co., Ltd., Ono Pharma Co. Ltd., Sumitomo Dainippon Pharma Co., Ltd., Nippon Boehringer Ingelheim Co. Ltd., Mitsubishi Tanabe Pharma Corp, Kissei Phama Co. Ltd., Takeda Pharma Co. Ltd., Daiichi Sankyo Co. Ltd., Sanofi K.K., MSD K.K., Sanwa Kagagu Kenkyusho CO., LtD., Astellas Pharma Inc., Kowa Pharma Co. Ltd., Abbott japan Co. Ltd., Kyowa Kirin Co., Ltd., Novo Nordisk Pharma Ltd., Eli Lilly Japan K.K., Tejin Pharma LtD., Nippon Chemiphar Co., Ltd., Johnson \& Johnson k.k. Medical Co., and Terumo Corp., and received honoraria from Taisho Pharma Co., Ltd., Ono Pharma Co. Ltd., Bayer Yakuhin, Ltd., Mitsubishi Tanabe Pharma Corp., Sanwa Kagaku Kenkyusho Co. Ltd., Astellas Pharma Inc., Sanofi K.K., Takeda Pharma Co. Ltd., MSD K.K., Kyowa Kirin Co. Ltd., Daiichi Sankyo Co. Ltd., Novo Nordisk Pharma Ltd., Kowa Pharma Co. Ltd., Sumitomo Dainippon Pharma Co. Ltd., Nippon Boehringer Ingelheim Co., Ltd., Eli Lilly Japan K.K., AstraZeneca K.K., Mochida Pharma Co. Ltd., Abbott japan Co. Ltd., Medtronic Japan Co. Ltd., Teijin Pharma Ltd., Arkray Inc., Kissei Pharma Co., Ltd., and Nipro Corp. outside the submitted work. The other authors have nothing to disclose.

\section{Author Contributions}

FT analyzed and interpreted the data, and wrote the manuscript. YH originated and designed the study, researched, analyzed and interpreted the data, and drafted the manuscript. AK and RS originated the study, researched and interpreted the data, and reviewed the manuscript. TO researched the data, and reviewed the manuscript. MH originated and designed the study, researched the data, and reviewed the manuscript. MF originated the study, researched and interpreted the data, and drafted the manuscript. All authors were involved in the writing of the manuscript and approved the final version of this article.

\section{References}

1. Yanase T, Yanagita I, Muta K, Nawata H (2018) Frailty in elderly diabetes patients. Endocr J 65: 1-11.

2. Marcell TJ (2003) Sarcopenia: causes, consequences, and preventions. J Gerontol A Biol Sci Med Sci 58: M911M916.

3. Landi F, Cruz-Jentoft AJ, Liperoti R, Russo A, Giovannini $\mathrm{S}$, et al. (2013) Sarcopenia and mortality risk in frail older persons aged 80 years and older: results from ilSIRENTE study. Age Ageing 42: 203-209.

4. Liu P, Hao Q, Hai S, Wang H, Cao L, et al. (2017) Sarco- penia as a predictor of all-cause mortality among community-dwelling older people: a systematic review and meta-analysis. Maturitas 103: 16-22.

5. Zhang X, Wang C, Dou Q, Zhang W, Yang Y, et al. (2018) Sarcopenia as a predictor of all-cause mortality among older nursing home residents: a systematic review and meta-analysis. BMJ Open 8: e 021252.

6. Tian S, Xu Y (2016) Association of sarcopenic obesity with the risk of all-cause mortality: A meta-analysis of prospective cohort studies. Geriatr Gerontol Int 16: 155- 
166.

7. Zhang X, Xie X, Dou Q, Liu C, Zhang W, et al. (2019) Association of sarcopenic obesity with the risk of allcause mortality among adults over a broad range of different settings: a updated meta-analysis. BMC Geriatr 19: 183.

8. Low S, Goh KS, Ng TP, Ang SF, Moh A, et al. (2020) The prevalence of sarcopenic obesity and its association with cognitive performance in type 2 diabetes in Singapore. Clin Nutr 39: 2274-2281.

9. Khadra D, Itani L, Tannir H, Kreidieh D, El Masri D, et al. (2019) Association between sarcopenic obesity and higher risk of type 2 diabetes in adults: a systematic review and meta-analysis. World J Diabetes 10: 311-323.

10. GBD Chronic Kidney Disease Collaboration (2020) Global, regional, and national burden of chronic kidney disease, 1990-2017: a systematic analysis for the Global Burden of Disease Study 2017. Lancet 395: 709-733.

11. Fox CS, Matsushita K, Woodward M, Bilo HJ, Chalmers $\mathrm{J}$, et al. (2012) Associations of kidney disease measures with mortality and end-stage renal disease in individuals with and without diabetes: a meta-analysis. Lancet 380: $1662-1673$

12. Wada T, Haneda M, Furuichi K, Babazono T, Yokoyama $\mathrm{H}$, et al. (2014) Clinical impact of albuminuria and glomerular filtration rate on renal and cardiovascular events, and all-cause mortality in Japanese patients with type 2 diabetes. Clin Exp Nephrol 18: 613-620.

13. Berhane AM, Weil EJ, Knowler WC, Nelson RG, Hanson RL (2011) Albuminuria and estimated glomerular filtration rate as predictors of diabetic end-stage renal disease and death. Clin J Am Soc Nephrol 6: 2444-2451.

14. Valmadrid CT, Klein R, Moss SE, Klein BE (2000) The risk of cardiovascular disease mortality associated with microalbuminuria and gross proteinuria in persons with older-onset diabetes mellitus. Arch Intern Med 160: 1093 1100.

15. Ida S, Kaneko R, Imataka K, Murata K (2019) Association between sarcopenia and renal function in patients with diabetes: a systematic review and meta-analysis. J Diabetes Res 2019: 1365189.

16. Bouchi R, Fukuda T, Takeuchi T, Minami I, Yoshimoto T, et al. (2017) Sarcopenia is associated with incident albuminuria in patients with type 2 diabetes: a retrospective observational study. J Diabetes Investig 8: 783-787.

17. Ramirez SP, McClellan W, Port FK, Hsu SI (2002) Risk factors for proteinuria in a large, multiracial, southeast Asian population. J Am Soc Nephrol 13: 1907-1917.

18. Sakai R, Hashimoto Y, Ushigome E, Miki A, Okamura T, et al. (2018) Late-night-dinner is associated with poor glycemic control in people with type 2 diabetes: The KAMOGAWA-DM cohort study. Endocr J 65: 395-402.

19. Kaji A, Hashimoto Y, Kobayashi Y, Sakai R, Okamura T, et al. (2019) Sarcopenia is associated with tongue pressure in older patients with type 2 diabetes: a cross-sectional study of the KAMOGAWA-DM cohort study. Geriatr Gerontol Int 19: 153-158.
20. Matsuo S, Imai E, Horio M, Yasuda Y, Tomita K, et al. (2009) Revised equations for estimated GFR from serum creatinine in Japan. Am J Kidney Dis 53: 982-992.

21. Haneda M, Utsunomiya K, Koya D, Babazono T, Moriya $\mathrm{T}$, et al. (2015) A new Classification of Diabetic Nephropathy 2014: a report from Joint Committee on Diabetic Nephropathy. J Diabetes Investig 6: 242-246.

22. Chen LK, Woo J, Assantachai P, Auyeung TW, Chou MY, et al. (2020) Asian Working Group for Sarcopenia: 2019 consensus update on sarcopenia diagnosis and treatment. J Am Med Dir Assoc 21: 300-307.e2.

23. Yoshimura Y, Wakabayashi H, Nagano F, Bise $T$, Shimazu S, et al. (2020) Sarcopenic obesity is associated with activities of daily living and home discharge in postacute rehabilitation. J Am Med Dir Assoc 21: 1475-1480.

24. Hashimoto Y, Hamaguchi M, Tanaka M, Obora A, Kojima T, et al. (2018) Metabolically healthy obesity without fatty liver and risk of incident type 2 diabetes: a meta-analysis of prospective cohort studies. Obes Res Clin Pract 12: 4-15.

25. Kanda Y (2013) Investigation of the freely available easyto-use software 'EZR' for medical statistics. Bone Marrow Transplant 48: 452-458.

26. Shafiee G, Keshtkar A, Soltani A, Ahadi Z, Larijani B, et al. (2017) Prevalence of sarcopenia in the world: a systematic review and meta-analysis of general population studies. J Diabetes Metab Disord 16: 21.

27. Ishii S, Chang C, Tanaka T, Kuroda A, Tsuji $\mathrm{T}$, et al. (2016) The association between sarcopenic obesity and depressive symptoms in older Japanese Adults. PLoS One 11: $\mathrm{e} 0162898$.

28. Yoo JH, Kim G, Park SW, Choi MS, Ahn J, et al. (2020) Effects of low skeletal muscle mass and sarcopenic obesity on albuminuria: a 7-year longitudinal study. Sci Rep 10: 5774 .

29. Nishida K, Hashimoto Y, Kaji A, Okamura T, Sakai R, et al. (2020) Creatinine/(cystatin $\mathrm{C} \times$ body weight) ratio is associated with skeletal muscle mass index. Endocr $J 67$ : 733-740.

30. Koliaki C, Liatis S, Dalamaga M, Kokkinos A (2019) Sarcopenic obesity: epidemiologic evidence, pathophysiology, and therapeutic perspectives. Curr Obes Rep 8: 458471.

31. Donini LM, Busetto L, Bauer JM, Bischoff S, Boirie Y, et al. (2020) Critical appraisal of definitions and diagnostic criteria for sarcopenic obesity based on a systematic review. Clin Nutr 39: 2368-2388.

32. Liao CD, Tsauo JY, Wu YT, Cheng CP, Chen HC, et al. (2017) Effects of protein supplementation combined with resistance exercise on body composition and physical function in older adults: a systematic review and metaanalysis. Am J Clin Nutr 106: 1078-1091.

33. Strychar I (2006) Diet in the management of weight loss. CMAJ 174: 56-63.

34. Sammarco R, Marra M, Di Guglielmo ML, Naccarato M, Contaldo F, et al. (2017) Evaluation of hypocaloric diet with protein supplementation in middle-aged sarcopenic 
obese women: a pilot study. Obes Facts 10: 160-167.

35. Kaji A, Hashimoto Y, Kobayashi Y, Wada S, Kuwahata M, et al. (2019) Protein intake is not associated with progression of diabetic kidney disease in patients without macroalbuminuria. Diabetes Metab Res Rev 35: e3150.

36. Jensen GL (2008) Inflammation: roles in aging and sarcopenia. JPEN J Parenter Enteral Nutr 32: 656-659.

37. Ziaaldini MM, Marzetti E, Picca A, Murlasits Z (2017) Biochemical pathways of sarcopenia and their modulation by physical exercise: a narrative review. Front Med (Lausanne) 4: 167.

38. Kalyani RR, Corriere M, Ferrucci L (2014) Age-related and disease-related muscle loss: the effect of diabetes, obesity, and other diseases. Lancet Diabetes Endocrinol 2: 819-829.

39. Sun L, Kanwar YS (2015) Relevance of TNF- $\alpha$ in the context of other inflammatory cytokines in the progression of diabetic nephropathy. Kidney Int 88: 662-665. 